\title{
Design and Implementation of Image Edge Detection Algorithm on FPGA
}

\author{
N. Shylashree ${ }^{1}$, M Anil Naik², A. S. Mamatha ${ }^{3}$, V. Sridhar ${ }^{4}$ \\ ${ }^{1,2}$ Department of Electronics and communication Engineering, RV College of Engineering, Bengaluru, India \\ ${ }^{3}$ Department of Electronics \& Communication Engineering, St. Joseph Engineering College, Mangaluru, \\ India \\ ${ }^{4}$ Department of Electronics \& Communication Engineering, Nitte Meenakshi Institute of Technology, \\ Bengaluru, India
}

Received: July 29, 2021. Revised: December 26, 2021. Accepted: January 14, 2022. Published: January 15, 2022.

\begin{abstract}
Image processing is an important task in data processing systems for applications such as medical sectors, remote sensing, and microscopy tomography. Edge recognition is a sort of image division method that is used to simplify the image records so as to reduce the amount of data to be processed. Edges are considered the most important in image processing because they are used to characterize the boundaries of an image. The performance of the Canny edge recognition algorithm remarkably surpasses the present edge recognition technology in various computer visualization methods. The main drawback of using Canny edge boundary is that it consumes lot of period due to its complex computation. In order to tackle this problem a hybrid edge recognition method is proposed in block stage to locate edges with no loss. It employs the Sobel operator estimate method to calculate the value and direction of the gradient by substituting complex processes by hardware cost savings, traditional non-maximum suppression adaptive thresholding block organization, and conventional hysteresis thresholding. Pipeline was presented to lessen latency. The planned strategy is simulated using Xilinx ISE Design Suite14.2 running on a Xilinx Spartan-6 FPGA board. The synthesized architecture uses less hardware to detect edges and operates at maximum frequency of $\mathbf{9 3 5}$ MHz.
\end{abstract}

Keywords-Edge Detection, Sobel, Canny, Hybrid, FPGA.

\section{INTRODUCTION}

Edges are significant local variations in the intensity of digital images. Edges are interpreted as a group of linked pixels that form boundary among two unlike sub regions. A way of segmenting a picture into isolated areas is a far widely used method in virtual picture processing including pattern identification, photograph morphology, and characteristic extraction. Edge detection permits users to distinguish significant changes in gray levels by observing features in the image. This texture marks the edge of the area and the start of other areas from the image, lessens the quantity of information in the picture and conserves the structural property of the picture.

Area detection entails a selection of mathematical operations finished on virtual pictures with the goal of specifying discrete factors that represent sharp adjustments in pixels that characterize the brightness and discontinuities of the image [1-3]. An edge is a group of squiggly lines knitted from pixels whose brightness changes rapidly in an image. Edge detection can be an elementary tool in image processing to notice features and characteristic extraction in individual applications according to machine and computer vision [4-6]. Edge detection assists in the optimization of network data measurements, assists in pattern recognition, and supports extraction of options [7]. Edge recognition can be a practice for judging breaks in gray-level pictures. Edges are one of the maximum crucial factors in image analysis. As an end result it plays a totally crucial position in a few applications of image processing, specific in machine vision [8-10].

There are various edge detection strategies, which include Prewitt, Robert, Sobel, and Laplace of Gaussian and Canny facet detector [11]. Amongst numerous aspect detection techniques, Canny detector presents improved overall effecting with the aid of casting off the constraints of all different aspect detectors, thereby reaching greater perfection in phrases of noise immunity and sharp boundaries [12-15]. In present applications, latency and computation charge are high for Canny side recognition algorithm. To trounce those flaws FPGA rooted computer hardware execution of hybrid area detection set of rules is preferred [16-20].

Applying canny edge detection circuitry to end-user cameras lowers hardware costs [21]. Previous implementations employed coarse calculations to avoid complex manipulation, reducing hardware costs, reducing input data corresponding to real-time applications and reducing accuracy. So, there is a compromise between the accuracy and the cost of the hardware [22-25]. These problems are resolved by introducing a new hybrid edge recognition algorithm with reasonable accuracy to reduce computing complexity. In order to reduce hardware cost and increase accuracy, the edge detection system adopts 
approximately methods, and pipeline technology is employed to reduce latency. A comparison shows the savings of logic cells at high throughput, with less hardware used by the developed hybrid edge detection system.

\section{EDGE DETECTION ALGORITHMS}

There are a number of techniques to achieve edge recognition. However, according to the mathematical operators used, they are also divided into two separate classes: 1. Gradient: The gradient approach detects edges by way of looking for the smallest exchange, including the most and minimum values of one pixel in a picture.

2. Laplacian: The Laplacian technique detects zero crosses and edges at the second spin-off of the image. It takes the secondary derivative from the image; therefore, the incomplete derivative operator is used to define the angle. Edges are distinguished in the following ways:

- Identify resident maxima or minima for the first derivative.

- Detecting the zero-crossing of the second derivative.

\section{A. Sobel Operator}

The Sobel operator is a classic first-order edge recognition operator that computes an estimate of the slope of the picture intensity. The edge precedes at the point where the gradation is the highest. The Sobel technique performs a $2 \mathrm{D}$ spatial gradient from the image, thus highlighting the high spatial frequency domain corresponding to the edge. It is commonly used to realize the estimated complete tilt size at every one point in $\mathrm{n}$ input gradation images [1].

\section{B. Prewitt Operator}

It is a Gradient-based operator, one of the best process to sense the direction and dimension of a picture. Calculates the slope of the picture intensity function to detect the edges of a picture. From the picture elements of a picture, the Prewitt operator produces a slope vector. Two 3 x 3 kernels convoluted with the input picture are used to compute the derivative calculation [1]

\section{Canny Edge Detection}

The Canny edge recognition approach is one of the standard edge recognition methods. Before finding the edges of a picture, it is very important to isolate the noise in the image and find the edges. Canny's method is a better way to apply severe values of tendencies and thresholds for later edges without interfering with the edge features of the image [2].

\section{METHODOLOGY}

\section{A. Canny edge detection algorithm}

An outline of traditional frame-based frugal edge recognition is shown in Fig. 1 This is a summary of the frugal edge recognition algorithm for an input picture of size $\mathrm{N} \mathrm{x} \mathrm{N}$, each in $\mathrm{n}$ bits. The fundamental steps for implementing a frugal edge recognition algorithm are described below.

1. Gradient in parallel and perpendicular directions, $f_{x}(x, y)$ and $f_{y}(x, y)$ respectively, for each picture element $(x, y)$ is calculated by performing convolution of the picture with gradient mask.

2. Magnitude and route of every picture element is computed. The importance and course outline the direction and strength of the threshold at each picture element.

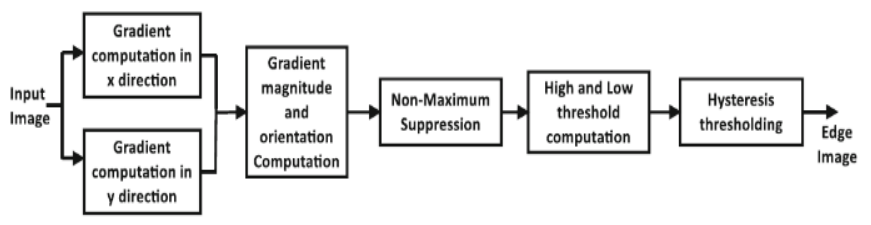

Figure 1. Implementation of Canny Edge Recognition Algorithm [2]

3. Non-Max suppression (NMS): Translates blurry edges of a picture to sharp edges and suppresses the minimum value (holds the local maximum value) in a gradient picture.

4. Threshold Computation: The possible edges are determined by the two thresholds; low and high and are calculated based on the whole picture tilt size histogram.

5. Hysteresis thresholding: Creates uninterrupted edge map by linking the values of gradient magnitude of each picture element with the two threshold values. It removes edge picture elements caused due to noise and illumination variation.

\section{B. Proposed Hybrid Edge Detection Algorithm}

The conventional Sobel and canny edge recognition algorithm have their own limitations when they are used individually. Sobel algorithm is applicable to simple picture but is not effective at recognizing edges in complex pictures. The Canny edge recognition algorithm is better than Sobel at recognizing edges at the cost of processing speed. The proposed edge recognition algorithm is used to overcome the limitations of Sobel and Canny edge recognition algorithm. In the proposed algorithm both Sobel and Canny algorithm are used together to improve the quality of edge recognition and the processing speed. In the proposed algorithm the color picture is converted into a grayscale picture and picture data is stored in two separate memory buffers, one buffer is for Canny algorithm and one buffer for Sobel algorithm. Horizontal pixel scanning is done using Canny algorithm and vertical pixel scanning is done using Sobel algorithm and both horizontal and vertical edge recognized pictures are combined to form efficient edge recognized picture.

There is no difference between level 1, 3 and level 5 of Canny algorithm and the Sobel algorithm, these levels are applied in block level. Gradient size and direction calculation in level 2 are estimated to lessen the price of hardware. Level 4, which is the two threshold computation termed adaptive threshold computation, is altered to refine the performance by manipulating the resident block statistics. For each picture in the record, non-overlying blocks are built and efficient classification techniques are used to classify (a) uniform areas (b) textures (c) middle edges and (d) strong edges.

Figure 2. Proposed Hybrid Edge Recognition Algorithm. 


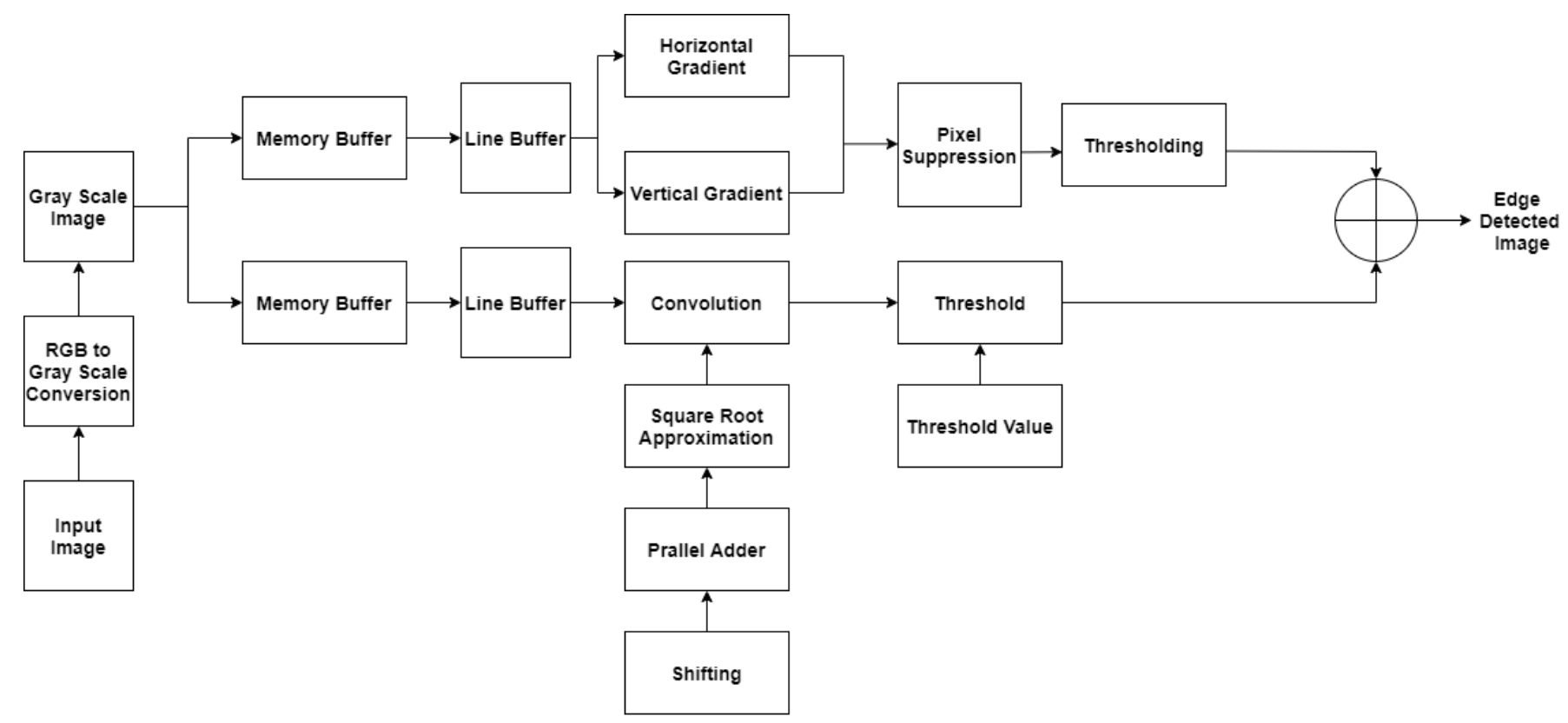

This classification technique uses a current pixel-centered $(3 \times 3)$ window to label pixels with uniform textures and edges using a local average of the magnitude of the slope of each pixel. Every block is then considered built on the total amount of edge picture elements. The two thresholds are calculated in record pictures are based on the median slope magnitude. The median of each block is implemented in a sliding window manner, computed using a histogram of the magnitude of the uniformly quantized gradient, improving the accuracy of the threshold on less hardware. Adaptive threshold computation aids to conquer unnecessary edges in even areas and find substantial edges in edge areas. Fig. 2 shows the summary of a new canny edge recognition design approach. A detailed explanation of the new hybrid edge recognition algorithm is as follows.

\section{1) RGB to Gray Scale conversion}

The input color picture is converted into gray scale picture. The color picture is split into red, green and blue planes and are multiplied with the threshold values to convert to the gray picture. Fig. 3 shows the structure of the RGB to grayscale conversion.

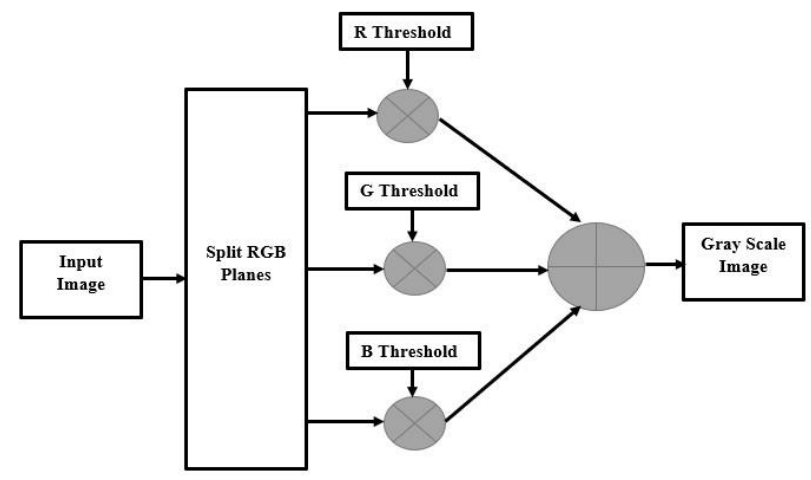

Figure 3. Structure of RGB to grayscale Conversion.

\section{2) Sobel operator based Gradient Computation}

A gradient mask is used for the Sobel operator. The reason for employing Sobel operator in place of other operators is because it integrates Gaussian smoothing and differentiation. Greater weight is assigned to picture element intensities around ends. Fig. 4 shows kernels of the Sobel operator in perpendicular and parallel direction.

\begin{tabular}{|l|l|l|}
\hline-1 & 0 & 1 \\
\hline-2 & 0 & 2 \\
\hline-1 & 0 & 1 \\
\hline
\end{tabular}

(a) Sobel operator kernel in $\mathrm{x}$ direction

\begin{tabular}{|c|c|c|}
\hline-1 & -2 & -1 \\
\hline 0 & 0 & 0 \\
\hline 1 & 2 & 1 \\
\hline
\end{tabular}

(b) Sobel operator kernel in y direction
Figure 4. Sobel Operator

For each $\operatorname{pixel}(x, y)$, the gradients in $x$ and $y$ direction denoted by $f_{x}(x, y)$ and $f_{y}(x, y)$ are calculated using (1) and (2).

$$
\begin{aligned}
& f_{x}(x, y)=\left(p_{2}-p_{0}\right)+\left(p_{5}-p_{0}\right)+\left(p_{0}-p_{6}\right) \\
& f_{y}(x, y)=\left(p_{6}-p_{0}\right)+\left(p_{7}-p_{1}\right)+\left(p_{0}-p_{2}\right)
\end{aligned}
$$

where $p 0=f(x-1, y-1), p 1=f(x . y-1)$, $p 2=f(x+1, y-1), p 3=f(x-1, y)$, $p 5=f(x+1, y), p 6=f(x-1, y+1)$, $p 7=f(x, y+1), p 8=f(x+1, y+1)$

In fact, identifying corners with high precision is not possible for Sobel operator. From the Equations (1) and (2), 2's complement subtraction is used to calculate the slope. The overflow bit (flog_x/flog_y) represents the sign of the resulting number. It is considered as positive value if overflow exists otherwise negative value. 2's complement is used to illustrate negative values. The architecture for the computation of $f_{x}(x, y)$ and $f_{y}(x, y)$ is shown in the Fig. 5. Shift-based multiplication and two's complement subtraction are used in proposed Canny edge detection to reduce hardware costs. 


\section{3) Gradient magnitude calculation}

Strength of an edge is defined by pixel size at specific pixel. Two square and one square root calculation is required as shown in equation 3. The size is performed using Eqn. 3,

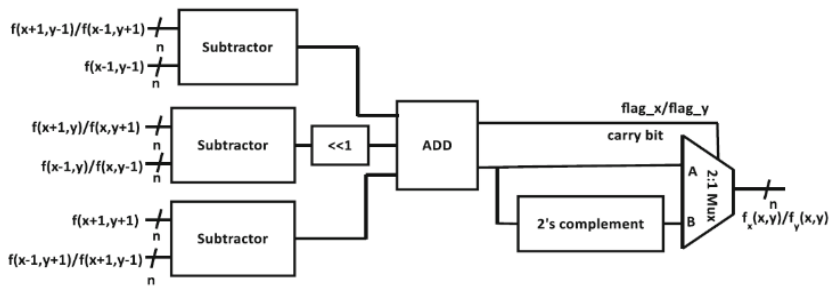

Figure 5. Architecture of $f_{x}(x, y)$ and $f_{y}(x, y)$

$$
\operatorname{mag}(x, y)=\sqrt{f_{x}(x, y)^{2}+f_{y}(x, y)^{2}}
$$

The cost paid to implement Eqn. 3 is very high hardware. Low-cost hardware technique called the square root approximation is proposed to calculate the $\operatorname{size} \operatorname{mag}(x, y)$. It is given by

$\operatorname{mag}(x, y)=\max ((0.875 a+0.5 b), a)$

Where $a=\max \left(f_{x}(x, y), f_{y}(x, y)\right)$ and

$b=\min \left(f_{x}(x, y), f_{y}(x, y)\right)$

In Eqn. 4 the multiplication operator is executed by shift block and the ADD block calculates the addition of the two inputs. Fig. 6 shows the square root approximation structure. Here, the CMP block compares an input value that produces a minimum and maximum value as output.

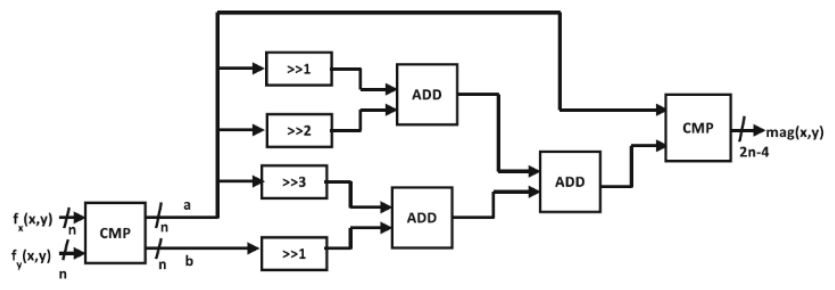

Figure 6. Structure of square root approximation

\section{4) Non-Max Suppression (NMS)}

It is a method used to thin the edges to maintain maxima beside the oblique direction of the image. As shown in Fig. 7, 8 contiguous pixels ( $3 \times 3$ windows) are needed to determine the maxima i.e., $\mathrm{g} 1, \ldots, \mathrm{g} 4$ and $\mathrm{g} 6, \ldots, \mathrm{g} 9$. The selector is used to find a picture element in the same direction as the current picture element portrayed as a and $b$ in Fig. 7. Current pixel g5 is compared with $\mathrm{a}$ and $\mathrm{b}$ using a comparator (CMP) to check for the presence of a maximum. If the maximum value exists for the current picture element, it is considered an edge picture element. It is suppressed if maximum value does not exist. The architecture of NMS is shown in Fig. 7.

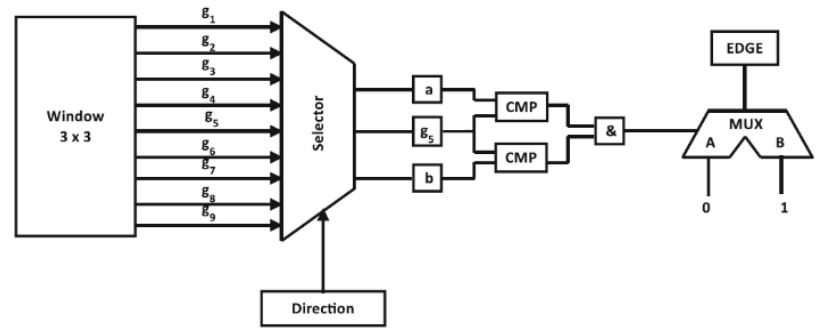

Figure 7. Structure of Non-Max Suppression

\section{5) Adaptive Threshold Computation}

The canny edge detector performance depends on the threshold. Therefore precise calculations are required. The median of the magnitude of the gradient is computed based on a uniformly quantized histogram of the magnitude of the gradient for each block type to help to find the threshold. Fig. 8 shows a schematic diagram of the histogram-based median filter structure, which is implemented in a sliding window method. Both the low and high thresholds are computed using a rule of thumb. The two threshold values are achieved by making use of median value as given in the Eqn. 4,

Low threshold $(T h L)=0.33 *$ Median Value

High threshold $(T h H)=1.66 *$ Median Value

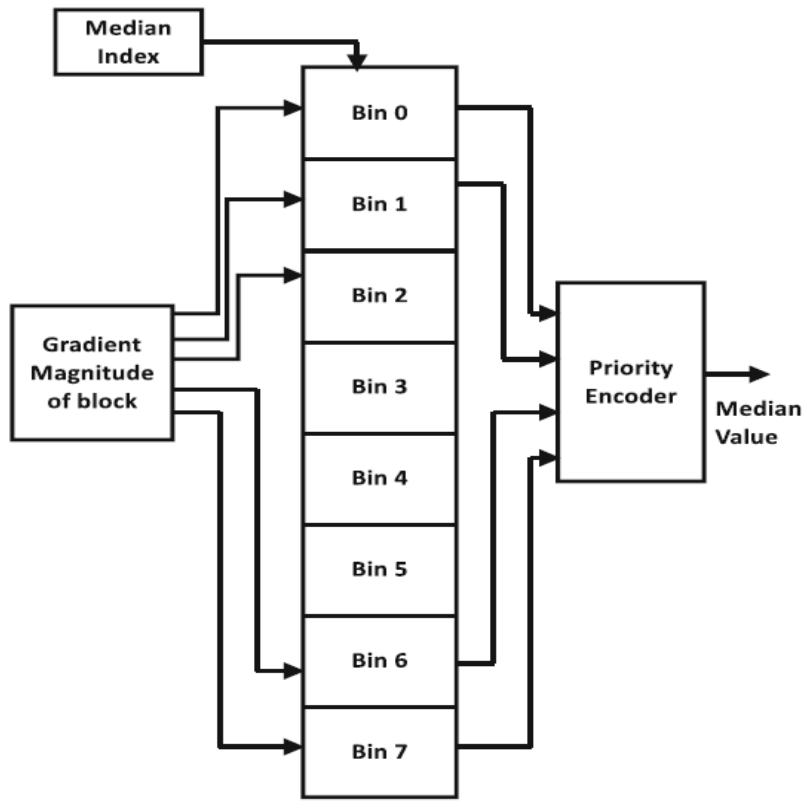

Figure 8. Structure of median filter based on Histogram.

Overall computation speed of the design is improved by employing a pipeline structure. It will breakdown the critical path. Every timing path will introduce a delay in the design, which path takes the maximum path is called as critical path. By using pipeline structure overall throughput of the design is increased. Fig. 9 shows the pipeline structure.

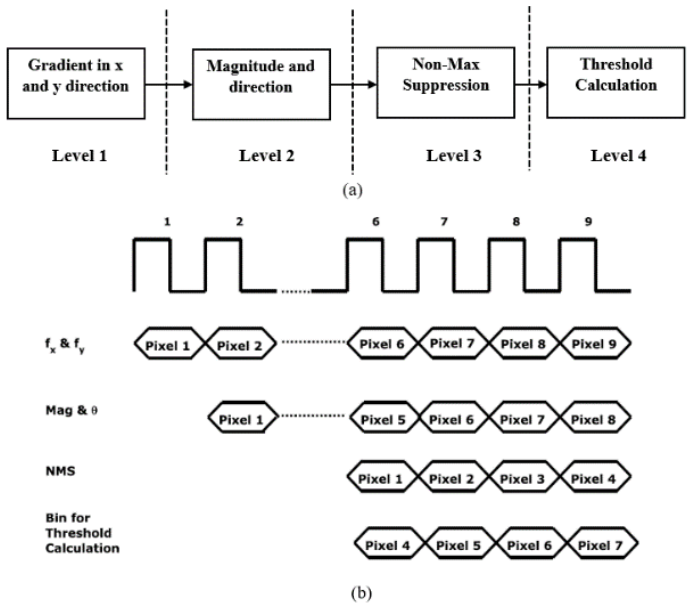

(b) 
Figure 9. Pipeline Structure

\section{IMPLEMENTATION}

\section{A. Implementation on MATLAB}

In this section, the proposed canny edge recognition algorithm is simulated using MATLAB tool. A MATLAB graphical user interface (GUI) application is developed to test the entire system. It is a MATLAB and Verilog co-simulation platform used to test the code. Fig. 10 shows the MATLAB GUI application, in the application the colour image will be read first and then the colour image is converted into grey scale image. The binary values of the image will be stored in the text files. The Verilog test bench reads the RGB pixel files and stores it in memory. This acts as the input to the image processing module.

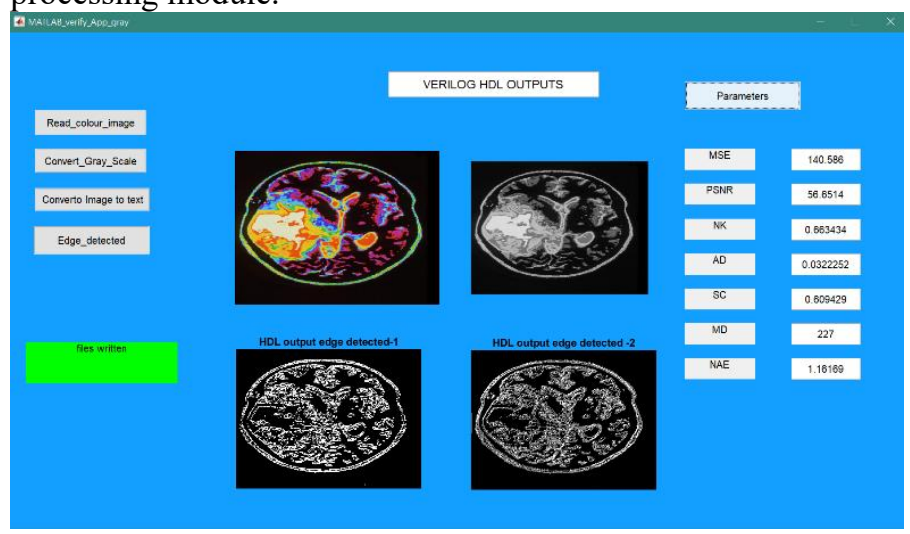

Figure 10. MATLAB GUI Application

\section{B. Implementation on XILINX}

The grey scale image data from MATLAB is sent to XILINX tool. Fig. 11 shows the memory in Verilog which has got the files from the text file.

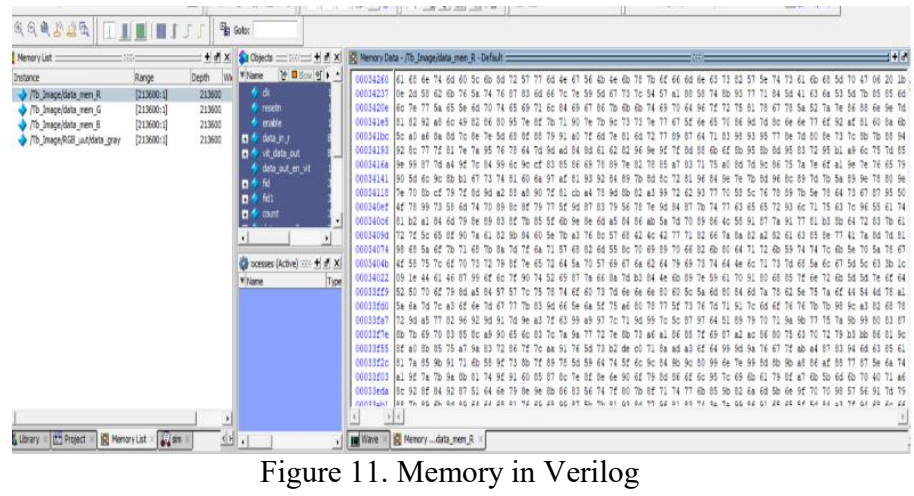

After running the Verilog test bench, it writes the output of gray scale, binary, edge and segmented data onto a memory and then onto a text file. Fig. 12 shows the edge detected data stored onto a memory. The MATLAB application will read output of Verilog and verify the algorithms and the edge detected image is displayed. The Verilog code is synthesized on a Spartan 6 FPGA. Fig. 13 shows the synthesized block view of the proposed hybrid edge detection algorithm.

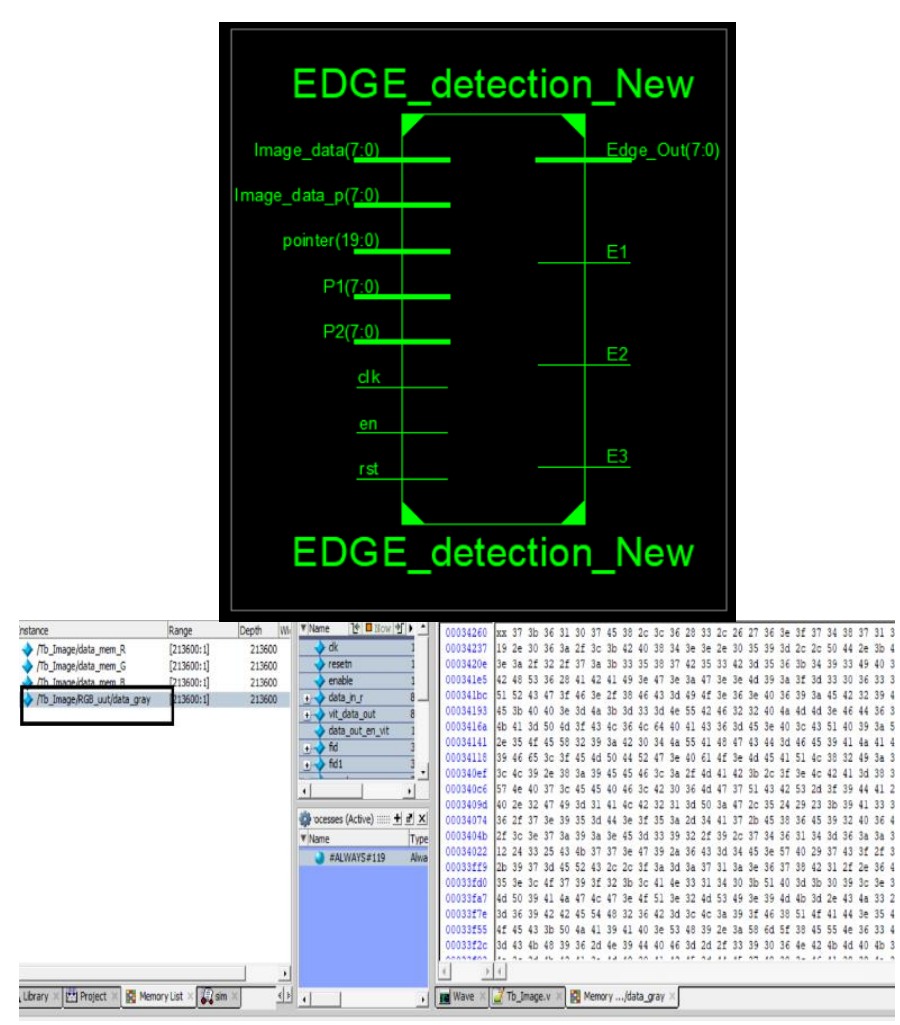

Figure 12. Edge detected data stored onto a memory.

Figure 13. Synthesized block view of proposed hybrid edge recognition algorithm

Fig. 14 shows the proposed RTL schematic of proposed Canny edge detection algorithm. The schematic diagram shows the different blocks of the proposed Canny edge recognition, it contains blocks like buffer, gradient, median filter, square approximation and thresholding. 


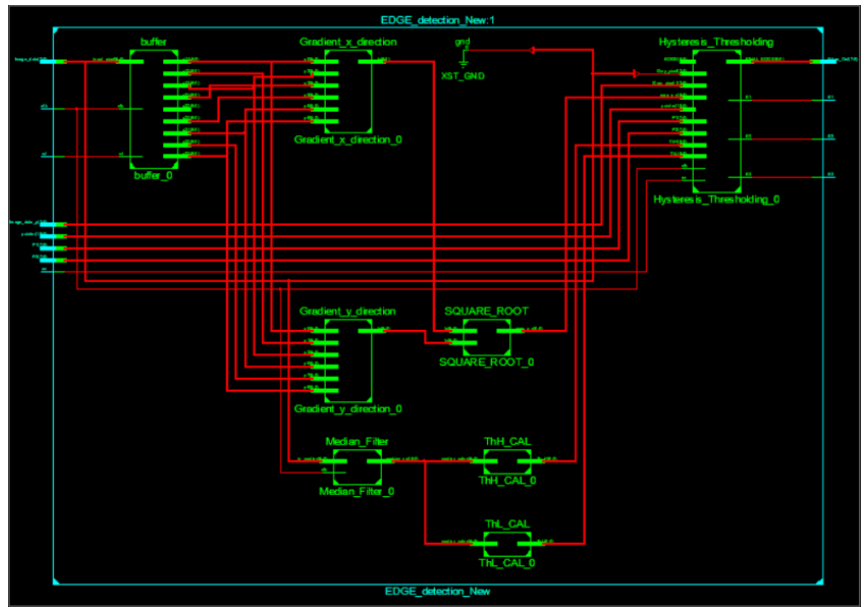

Figure 14. RTL Diagram of Hybrid Edge Recognition Algorithm

\section{V.RESULTS AND DISCUSSION}

The simulation of proposed algorithm for hybrid edge recognition is tested on colour picture of any size, these pictures are converted into gray scale and resized to $512 \times 512$ standard size. algorithm. Computation of gradient direction and magnitude using Approximation method, uniform quantization method for calculation of median value which is achieved in gliding window manner, which shows better results than traditional Canny and Sobel algorithms. Table 1 shows the image parameters such as PSNR, MSE, average difference, maximum difference and structural content of the picture. The MSE joins debasement work and statistical characteristics of commotion within the edge detected picture. MSE specifies the normal distinction of the picture elements all through the original ground truth picture with edge recognized picture. The higher MSE demonstrates a more noteworthy distinction between the unique and prepared picture. MSE should be higher in picture edge recognition to ensure that it found more edge points on the picture and also it is capable to detect weak edge points. PSNR is one of the blunder measurements utilized in picture compression. Based on picture compression, PSNR is utilized to degree the quality of reconstructed picture of Lossy and Lossless compression. Higher PSNR implies more commotion evacuated.

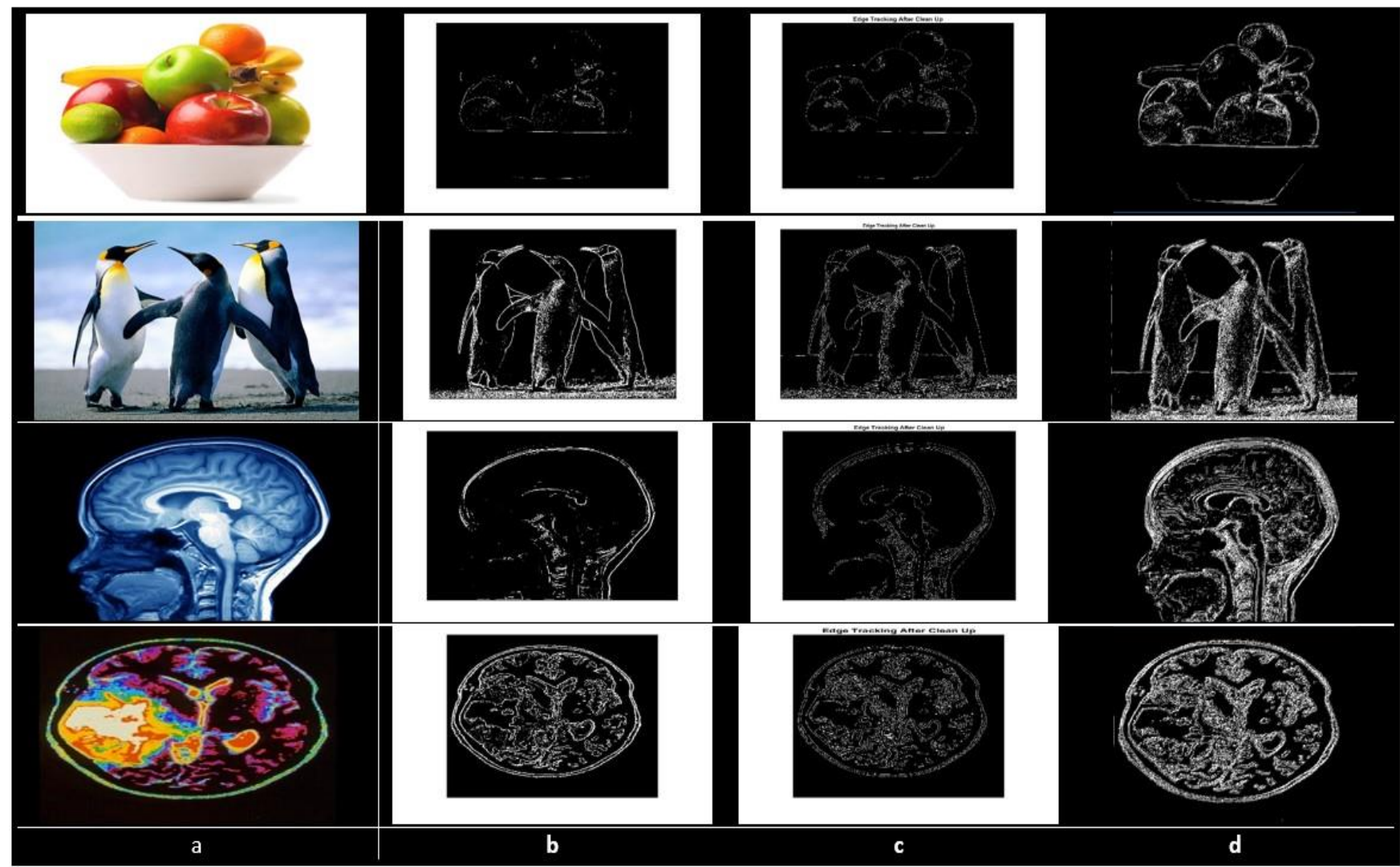

Figure 15. Edge detection a) Original Picture b) Sobel edge recognition, c) Canny edge recognition d) Hybrid edge recognition algorithm.

The simulation of proposed algorithm for edge recognition is executed using Xilinx Design Suite. The strategy is verified on Xilinx Spartan-6, Virtex-6 and Zynq FPGA board. Fig. 15 shows the comparison results of Sobel, Canny and proposed
Table 1. Summary Of Parameters

\begin{tabular}{|c|c|c|c|c|}
\hline Parameters & Image 1 & Image 2 & Image 3 & Image 4 \\
\hline $\begin{array}{c}\text { Mean } \\
\text { Square } \\
\text { Error }\end{array}$ & 704.258 & 441.408 & 165.355 & 140.586 \\
\hline $\begin{array}{c}\text { Peak Signal } \\
\text { to Noise }\end{array}$ & 49.6535 & 51.6824 & 55.9466 & 56.6514 \\
\hline
\end{tabular}




\begin{tabular}{|c|c|c|c|c|}
\hline Ratio & & & & \\
\hline $\begin{array}{c}\text { Average } \\
\text { Difference }\end{array}$ & 0.756412 & 0.418653 & 0.0874878 & 0.0322252 \\
\hline $\begin{array}{c}\text { Structural } \\
\text { Content }\end{array}$ & 14.0774 & 2.79064 & 0.879958 & 0.609429 \\
\hline $\begin{array}{c}\text { Maximum } \\
\text { Difference }\end{array}$ & 255 & 255 & 255 & 227 \\
\hline
\end{tabular}

proposed Canny edge recognition algorithm utilizes less computer hardware compared to the traditional Sobel and Canny algorithm. The proposed design is simulated on different target boards and the results shows that the proposed algorithm consumes less time to detect edges. Pipelined architecture helps to increase the overall speed. The proposed design is able to detect the image edges at $935 \mathrm{MHz}$ of operating frequency on Xilinx Virtex-6 board. The proposed

Table 2 shows the device utilization summary of Sobel, Canny and the proposed algorithm. Observation shows that the

Table 2. Device Utilization Summary

\begin{tabular}{|c|c|c|c|c|c|c|}
\hline $\begin{array}{c}\text { Edge Recognition } \\
\text { Methods }\end{array}$ & Image Size & FPGA Device & Used Slices & No. of LUTs & $\begin{array}{c}\text { Frequency } \\
(\mathbf{M H z})\end{array}$ & Time \\
\hline Sobel Edge Detection & $512 \times 512$ & Spartan 6 & 0 & 88 & 100 & $10 \mathrm{~ns}$ \\
\hline Canny Edge Detection & $512 \times 512$ & Xilinx Virtex-5 & $17928 / 28495$ & - & 272 & $0.13 \mathrm{~ms}$ \\
\hline Proposed Algorithm & $512 \times 512$ & Spartan 6 & $104 / 93296$ & $302 / 46648$ & 549 & $1.82 \mathrm{~ns}$ \\
\cline { 3 - 7 } & & Xilinx Viretx-6 & $104 / 93120$ & $302 / 46560$ & 724 & $1.38 \mathrm{~ns}$ \\
\cline { 3 - 7 } & & Zynq & $104 / 157200$ & $302 / 78600$ & 935 & $1.06 \mathrm{~ns}$ \\
\hline
\end{tabular}

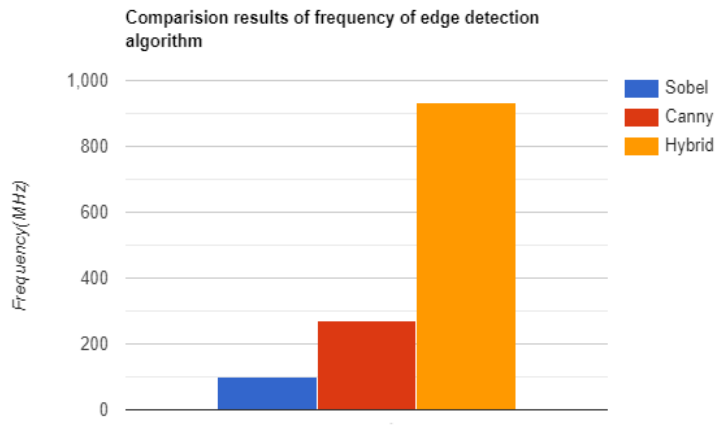

Edge Detection Algorithm

Figure 16. Comparison result of frequency of edge detection algorithms.

Fig. 16 shows comparison between the operating frequency of the Sobel, canny and the proposed hybrid edge recognition algorithms. From the graph it is clearly evident that the hybrid architecture is faster than the convention algorithms.

\section{CONCLUSION}

Pipeline technology is used in proposed Canny edge detection algorithm for simultaneous multi-block processing, so there is increase in processing speed. The method used to compute the direction and magnitude of the gradient is the gliding window method in the median filter based on histogram structure, under which hardware is underutilized. Results shows that the proposed algorithm consumes less resource in comparison to the conventional Sobel and Canny algorithm and it requires only $1.06 \mathrm{~ns}$ to detect the edges of $512 \times 512$ picture and is operating at a maximum frequency of $935 \mathrm{MHz}$.

\section{REFERENCES}

[1] Dr. R. Menaka, Dr. R. Janarthanan, Dr. K. Deeba, "FPGA implementation of low power and high-speed image edge detection algorithm", Journal of Microprocessors and Microsystems, Volume 75, Article 103053, ELSEVIER June 2020.

[2] D. Sangeetha, P. Deepa, "FPGA implementation of costeffective robust Canny edge detection algorithm" Journal of Real Time Image Processing, Springer Link Mar 2016.

[3] Kanopouls, N., Vasanthavada, N., Baker, R.L.: Design of an image edge detection filter using the sobel operator. IEEE J. Solid-State Circuits 23(2), 358-367 (1988)

[4] Qian Xu, Srenivas Varadarajan, Chaitali Chakrabarti, Lina J. Karam, "A Distributed Canny Edge Detector: Algorithm and FPGA Implementation", IEEE Transactions on Image Processing Volume 23 Issue 7, 2014, Pages:2944-2960.

[5] Li Xuan, Zhang Hong, "An improved canny edge detection algorithm", 8th IEEE International Conference on Software Engineering and Service Science (ICSESS), ISSN: 2327-0594, 2017, DOI:10.1109/ICSESS.2017.8342913.

[6] Jair Trapé Goulart, Rosana Almada Bassani, José Wilson Magalhães Bassani, "Application based on the Canny edge detection algorithm for recording contractions of isolated cardiac myocytes", Computers in Biology and Medicine, Volume 81, Pages 106-110,2017.

[7] Ms. Pawar P. H, Prof. Patil R. P, "Image Edge Detection Techniques using MATLAB Simulink", International Journal of Engineering Research \& Technology (IJERT), Volume 3 Issue 6, 2014.

[8] Miroslav Hagara, Radovan Stojanovi, TomášBagala, Peter Kubinec, "Grayscale image formats for edge 
detection and for its FPGA implementation", Journal of Microprocessors and Microsystems Volume 75, Article 103056, ELSEVIER, 2020.

[9] Soumen Santra, Surajit Mandal, Kunal Das, Joy Bhattacharjee, Avijit Roy, "A Modified Canny Edge Detection Approach to Early Detection of Cancer Cell", 2019 3rd International Conference on Electronics, Materials Engineering \& Nano-Technology, DOI: 10.1109.

[10] Girish Chaple, R. D. Daruwala, "Design of Sobel Operator based Image Edge Detection Algorithm on FPGA", International Conference on Communication and Signal Processing, April 3-5, 2014.

[11]Juseong Lee, Hoyoung Tang, Jongsun Park, "Energy Efficient Canny Edge Detector for Advanced Mobile Vision Applications", IEEE Transactions on Circuits and Systems for Video Technology, Volume 8, Issue 4, Page no-1037-1046,2016.

[12] Mamta Joshi, Ashutosh Vyas, "Comparison of Canny edge detector with Sobel and Prewitt edge detector using different image formats", International Journal of Engineering Research \& Technology (IJERT) ISSN: 2278-0181.

[13] Hu Jing, Xing Xiaoqiong, "Sports image detection based on FPGA hardware system and particle swarm algorithm", Journal of Microprocessors and Microsystems Volume 75, Article 103348, ELSEVIER, 2020.

[14] Shivani, Er. Harjeet Singh, "The Performance Analysis of Edge Detection Algorithms for Image Processing Based on Improved Canny Operator", International Journal of Computer Trends and Technology, Volume 68, Issue 10, Page no-29-34, 2020.

[15] M. M. Mehdy, P. Y. Ng, E. F. Shair, N. I. Md Saleh, and C. Gomes, "Artificial Neural Networks in Image Processing for Early Detection of Breast Cancer", Computational and Mathamatical Methods in Medicine 2017; 2017: 2610628.Published online 2017 Apr 3.

[16]Yuan-Kai Huo, G. Wei, Y. D. Zhang and L. N. Wu, "An adaptive threshold for the Canny Operator of edge detection",2010 International Conference on Image Analysis and Signal Processing, Zhejiang, 2010, pp. 371374.

[17]Cai-Xia Deng, Gui-Bin Wang and Xin-Rui Yang,"Image edge detection algorithm based on improved Canny operator," 2013 International Conference on Wavelet Analysis and Pattern Recognition, Tianjin, 2013, pp. 168-172.

[18]Md Khurram Monir Rabby, Brinta Chowdhury, Jung H. Kim, "A Modified Canny Edge Detection Algorithm for Fruit Detection \& Classification", 10th International Conference on Electrical and Computer Engineering (ICECE), Page no. 237-240, 2018.

[19]Marina Nikolic, Eva Tuba, Milan Tuba, "Edge detection in medical ultrasound images using adjusted Canny edge detection algorithm", 2016 24th Telecommunications Forum (TELFOR), 22-23 Nov. 2016, Belgrade, Serbia.

[20]Chia-Jui Hsieh, Ta-Ko Huang, Tung-Han Hsieh, "Compressed sensing based CT reconstruction algorithm combined with modified Canny edge detection", Physics in Medicine \& Biology, Volume 63, Number 15, 27 July 2018.

[21]Samuel H. Chang, Leiguang Gong, Maoqing Li, Xiaoying $\mathrm{Hu}$, Jingwen Yan, "Small retinal vessel extraction using modified Canny edge detection", 2008 International Conference on Audio, Language and Image Processing, 7-9 July 2008, Shanghai, China.

[22]Zorana Stosic, Petar Rutesic, "An Improved Canny Edge Detection Algorithm for Detecting Brain Tumors in MRI Images", International Journal of Signal Processing, Volume 3, 2018.

[23]Dr. Abdulsattar M. Khidhir, Nawal Younis Abdullah, "FPGA Based Edge Detection Using Modified Sobel Filter", International Journal for Research and Development in Engineering (IJRDE), Volume 2 Issue 1, 2013, Pages: 22-34.

[24]E.J. Mohammad, M. JawadKadhim, W.I. Hamad, S.Y. Helyel, "Study sobel edge detection effect on the image edges using matlab", Int. J. Innov. Res. Sci. Eng. TechVolume- 3, Issue 3, March 2014.

[25]P. Shanmugavadivu, Ashish Kumar, "Modified EightDirectional Canny for Robust Edge Detection", 2014 International Conference on Contemporary Computing and Informatics (IC3I), 27-29 Nov. 2014, Mysore, India.

Dr. Shylashree $\mathbf{N}$ is currently working as Associate Professor in the Department of Electronics and Communication Engineering at RV College of Engineering, Bengaluru. She is having 15 years of teaching experience. She was a recipient of the best $\mathrm{PhD}$ thesis award for the year 2016-2017 in Electronics and Communication Engineering from BITES. She has received the best IEEE researcher award in IEEE-AGM meeting held during 2021 from Bangalore IEEE section. She has also received the best paper award in IEEEICERECT held during 2015 at Mandya. She has research publication in 18 International Journals (out of which 5 journals are Springer-SCI journals), 4 Springer book chapters and 9 International conferences. She has published 2 patents and filed 1 German patent in the area of cryptography. She has also published 2 patents in the area of VLSI out of which one of them got a grant. She is also the author of the Network Theory textbook, Engineering Statistics \& Linear Algebra text book and Control Engineering textbook. She has funded project on chalcogenide materials \& consultancy projects on FPGA and has delivered many technical talks on VLSI. She has delivered lectures as a subject matter expert in VTU eshikshana and EDUSAT program. She is a recipient of international travel grant under SERB young research scholar category. She is a life member of ISTE, IETE, fellow member of ISVE, Senior member of IEEE and IEEE-CAS Bangalore section execom member.

Email- shylashreen@rvce.edu.in, shylashashi@gmail.com

Mr. Anil Naik M is a post graduate student of VLSI Design and Embedded systems. He is currently pursuing his final year in Master of Technology degree at RV College of Engineering, Bengaluru, India. His areas of interest are VLSI, RTL design and Physical Design. Email- anilnaikm.lvs19@rvce.edu.in 
Dr. Mamatha A.S (Corresponding Author) is currently working as Associate Professor in the Department of Electronics and Communication Engineering at St. Joseph Engineering College, Mangalore. She has 23 years of teaching experience. She is the author of four international journals and six international conferences in the field of Multispectral Image Compression. She is the author of the Network Theory textbook, Engineering Statistics \& Linear Algebra textbook and Control Engineering text book. Her areas of interest are Signal Processing, Image Compression, and Control Engineering etc. She is a Senior IEEE member.

Email-mamathatanya@gmail.com, mamathag@sjec.ac.in

Dr. V Sridhar is currently working as Emeritus Professor in the Department of Electronics and Communication Engineering at Nitte Meenakshi Institute of Technology, Bengaluru. He has done his Ph.D from IIT Delhi and postdoctoral research from Uniten University, Malaysia. He has 35 years of teaching experience. He has served as an acting Vice-Chancellor and Registrar (Evaluation) in VTU during 2016 and 2004 respectively; He has worked as Principal in P.E.S College of engineering Mandya, during 2008 to 2018. He has guided $11 \mathrm{PhD}$ students and currently he is also guiding a $\mathrm{PhD}$ student. He has research publication in more than 50 international journals and conferences. He has successfully completed many funded research projects and delivered more than 50 technical talks across India and Abroad. He has published 2 patents in the field of Cryptography. He is the author of the Network Theory, Engineering Statistics \& Linear Algebra and Control Engineering textbook. He is an IEEE senior member and life member of ISTE, MBMSI \& FIETE.

His areas of interest are Bio-medical signal processing, VLSI Design, Cryptography \& Network security, wireless communication and ergonomics.

Email-venusridhar@yahoo.com, dean-academics@nmit.ac.in

\section{Creative Commons Attribution License 4.0 (Attribution} 4.0 International, CC BY 4.0)

This article is published under the terms of the Creative Commons Attribution License 4.0

https://creativecommons.org/licenses/by/4.0/deed.en_US 